\title{
VARIATIONAL COMPLETENESS FOR COMPACT SYMMETRIC SPACES
}

\section{ROBERT HERMANN ${ }^{1}$}

We prove the following theorem: Let $K$ be a connected symmetric subgroup of the group of isometries of a compact connected globally Riemannian symmetric space $M$. Then, $K$ acts in a variationally complete manner on $M$. (We refer to the work of R. Bott and H. Samelson $[1 ; 2]$ for a definition of this concept and applications to topology.)

Let $G$ be a compact, connected Lie group, $L$ a connected symmetric subgroup of $G$. Bott and Samelson have proved the following: (1) $L$ acts in a variationally complete manner on $G / L$, the right coset space, (2) $L$ acts via the linear isotropy representation in a variationally complete way on the tangent space at a point of $G / L$.

Our theorem then generalizes (1), but does not imply (2). The method is essentially the same as Bott's [1], but uses a Lie algebra approach in a stronger way. The proof then provides an exposition of Bott's very important result from a Lie-algebra point of view. Notice also that it suggests the general program of studying the Jacobi-fields on a symmetric space as a problem of Lie algebra theory, even in the nonvariational complete case.

1. We briefly recall Bott's notations: If $p \in M, M_{p}$ denotes the tangent space to $M$ at $p$. Let $O_{p}(K)$ be the orbit of $K$ at $p$, let $O(K)_{p}$ be its tangent space at $p$, and let $g: R \rightarrow M$ be a geodesic of $M$ beginning at $p$, perpendicular to $O_{p}(K)$. Consider the vector space $J_{\theta}$ of Jacobi vector fields along $g$. A Jacobi field is a map $t \rightarrow Y_{t} \in M_{g}(t)$ for $t \in R$ that is a solution of the Jacobi Equation:

$$
Y_{t}^{\prime \prime}+R\left(X_{t}, Y_{t}\right)\left(X_{t}\right)=0,
$$

where $t \rightarrow X_{t}$ is the tangent vector field to the geodesic $g, R\left(X_{t}, Y_{t}\right)$ is the linear map $M_{g(t)} \rightarrow M_{g(t)}$ defined by the curvature form evaluated at $\left(X_{t}, Y_{t}\right)$ and $t \rightarrow Y_{t}^{\prime \prime}$ is the second covariant derivative of the field $Y_{t}$ along $g$.

$J_{0}^{K}$ denotes the focal subspace of $J_{0}$ relative to $O(K)$, i.e. $J_{0}^{K}$ consists of those fields $Y_{t}$ satisfying the initial conditions

Received by the editors December 18, 1958.

1 This research was supported in part by OOR, U. S. Army under contract no. Da19-020-ORD-3778. 


$$
\begin{gathered}
Y_{0} \in O(K)_{p}, \\
Y_{0}^{\prime}+T_{\theta}\left(Y_{0}\right) \in O(K)_{p}^{\perp}, \text { where } T_{\theta} \text { is a certain linear }
\end{gathered}
$$

transformation $O(K)_{p} \rightarrow O(K)_{p}$.

Hence, $\operatorname{dim} J_{a}^{K}=\operatorname{dim} M$.

Let $K$ denote the Lie algebra of $K$. There is a linear mapping $\pi: K \rightarrow J_{g}^{K}$ such that for $k \in K, \pi(k)$ is obtained by restricting the vector field determined by $k$ on $M$ to $g$.

By definition, to prove variational completeness we must show that every element of $J_{0}^{K}$ that vanishes at some point of $g$ must lie in $\pi(K)$.

2. $M$ is a coset space $G / L$, where $L$ is a compact, symmetric subgroup of a compact, connected Lie group $G$. It evidently suffices to prove the theorem in the case where $p$ is the identity coset.

We have a natural reduction $G=L \oplus M$, with $[M, M] \subset L$ and $[L, M] \subset M$. (For the ideas and results of the differential geometry of symmetric spaces, see [3].) $M_{p}$ can be identified with $M$, and each $M_{o(t)}$, for $t \in R$, can be identified with $M_{p}$ by parallel transport along $g$. There is then a correspondence between vector fields along $g$ and curves $t \rightarrow Y_{t}$ in $M$. The metric on $M$ can be considered as induced by a positive definite quadratic form on $G$ invariant under Ad $G$.

Let $P$ denote the projection of $G$ on $M$. Then $N=P(K)$ is identified with $O(K)_{p}$. Let $X \in M$ correspond to $X_{0}$. Then $X \in N^{\perp}$. One sees that a curve $t \rightarrow Y_{t}$ in $\boldsymbol{M}$ corresponds to a vector field in $J_{0}^{K}$ if and only if

$$
\begin{aligned}
& Y_{0} \in N, \\
& Y_{0}^{\prime}+T_{\theta}\left(Y_{0}\right) \in N \perp, \\
& Y_{\iota}^{\prime \prime}=(\operatorname{Ad} X)^{2} Y_{t},
\end{aligned}
$$

where $t \rightarrow Y_{t}^{\prime}$ is now the derivative in the ordinary sense. (One uses the explicit formula $\operatorname{Ad}[x, y]=-R(x, y)$ for the curvature in symmetric spaces and the fact that curvature is invariant under parallel translation in identifying $R\left(X_{t}, Y_{t}\right) X_{t}$ with $(\operatorname{Ad} X)^{2} Y_{t}$, [3].)

If $k \in K, \pi(k)$ corresponds to the curve $t \rightarrow \pi(k)_{t}$ $=P((\operatorname{Ad} \operatorname{Exp} t X)(k))$, hence $\pi(k)_{0}=P(k), \pi(k)_{0}^{\prime}=P([X, k])$.

The question of variational completeness can now be treated in this Lie algebra setting as a property of solutions of vector-valued ordinary linear differential equations (2.2) with constant coefficients. In particular, a reduction of $\boldsymbol{M}$ into subspaces invariant under 
$(\operatorname{Ad} X)^{2}$ leads to a decomposition of solutions of (2.2) into solutions taking values in the invariant subspaces.

3. The proof.

(a) kernel $\pi=K \cap L \cap$ kernel Ad $X$.

(b) $\operatorname{dim} \pi(\boldsymbol{K})=\operatorname{dim} \boldsymbol{K}-\operatorname{dim} \boldsymbol{K} \cap L \cap$ kernel Ad $X=\operatorname{dim} P(\boldsymbol{K})$ $+\operatorname{dim} K \cap L-\operatorname{dim} K \cap L \cap$ kernel $\quad$ Ad $X=\operatorname{dim} P(K)$ $+\operatorname{dim} \operatorname{Ad} X(K \cap L)$.

(c) $\left[K^{\perp}, K^{\perp}\right) \subset K,\left[K, K^{\perp}\right] \subset K^{\perp}$, since $K$ is a symmetric subalgebra of $G$. (The perpendicular operation $\perp$ is always with respect to the given metric on $\boldsymbol{G}$.) $L^{\perp}=\boldsymbol{M}$. $(\operatorname{Ad} X)^{2}(P(\boldsymbol{K})) \subset P(\boldsymbol{K})$, since $X \in K^{\perp} \cap L^{\perp}$.

(d) Ad $X(K \cap L) \subset K^{\perp} \cap L^{\perp}$ hence Ad $X(K \cap L) \subset P(K)^{\perp} \cap M$. $(\operatorname{Ad} X)^{2}(\operatorname{Ad} X(K \cap L)) \subset(\operatorname{Ad} X)^{2}\left(K^{\perp} \cap L^{\perp}\right) \subset \operatorname{Ad} X(K \cap L)$.

(e) Define $\boldsymbol{Q}=\boldsymbol{M} \cap P(\boldsymbol{K})^{\perp} \cap \operatorname{Ad} X(\boldsymbol{K} \cap \boldsymbol{L})^{\perp}=(P(\boldsymbol{K})+\operatorname{Ad} X(\boldsymbol{K} \cap \boldsymbol{L}))^{\perp}$ $\cap M$. Then, $(\operatorname{Ad} X)^{2}(Q) \subset Q, Q \subset K^{\perp} \cap L^{\perp}$.

(f) $\operatorname{Ad}^{2} X(Q)=0$, for, Ad $X(Q) \subset K \cap L$, hence $\operatorname{Ad}^{2} X(Q)$ $\subset \operatorname{Ad} X(K \cap L) \cap Q=0$.

(g) $\operatorname{Dim} M=\operatorname{dim} \boldsymbol{M}=\operatorname{dim} J_{\theta}^{K}=\operatorname{dim}(\pi(K)+\boldsymbol{Q})$.

(h) If $k \in K$, then $\pi(k)_{t} \in Q^{\perp}$ for all $t \geqq 0$.

Proof. $\pi(k)_{t}=P(\sinh (\operatorname{Ad} t X)(k))+P(\cosh (\operatorname{Ad} t X)(k))$. Now, $P(\cosh (\operatorname{Ad} t X)(k)) \in P(K) \subset Q^{\perp}$. Then, $\sinh (\operatorname{Ad} t X)(k) \in Q^{\perp}$ because of (e) and (f), and the fact that $(\operatorname{Ad} X)^{2}$ is a symmetric transformation, with respect to the positive-definite quadratic form on $G$, that commutes with $P$.

Now, define a map $\psi: \boldsymbol{Q} \rightarrow J_{g}^{K}$ as follows: For $\boldsymbol{q} \in \boldsymbol{Q}, \psi(\boldsymbol{q})_{t}$ is the curve in $\boldsymbol{M}$ satisfying (2.2) and

$$
\begin{aligned}
\psi\left(\boldsymbol{q}_{0}\right) & =0, \\
\psi\left(\boldsymbol{q}_{0}^{\prime}\right) & =\boldsymbol{q} .
\end{aligned}
$$

Because of (e) and (f), $\psi(q)_{t}=t \boldsymbol{q}$, and hence $\psi$ is one-to-one. Then $J_{0}^{K}=\psi(Q)+\pi(K), \psi(Q) \cap \pi(K)=0$ and the theorem follows. For if $t \rightarrow Y_{t}$ is an element of $J_{\theta}^{K}, Y_{t}=\pi(k)_{t}+t q$ for $k \subset K, q \in Q$, and $Y_{t}=0$ for some $t>0$, then $q=0$ by (h).

\section{BIBLIOGRAPHY}

1. R. Bott, An application of the Morse theory to the topology of Lie groups, Bull. Soc. Math. France vol. 4 (1956) pp. 251-281.

2. R. Bott and H. Samelson, Applications of the theory of Morse to symmetric spaces, Amer. J. Math. vol. 80 (1958) pp. 964-1029.

3. K. Nomizu, Invariant affine connections on homogeneous spaces, Amer. J. Math. vol. 76 (1954) pp. 33-65.

HARVARD UNIVERSITY 